\title{
Supply Chain Risk Management in Chinese Chemical Industry based on Stochastic Chance-Constrained Programming Model
}

\author{
Liping Liu, Shuxia $\mathrm{Li}^{*}$ and Yifan Wu \\ Research Center of Virtual Business, Institute of Operations and Supply Chain Management, School of Business, East China University \\ of Science and Technology, 130 Meilong Road, Shanghai, 200237, P. R. China
}

Received: 4 Jun. 2013, Revised: 9 Oct. 2013, Accepted: 10 Oct. 2013

Published online: 1 May. 2014

\begin{abstract}
It is necessary to research on how to manage risks in Chinese chemical industry supply chain effectively. After analyzing supply chain risks and mitigation strategies in Chinese chemical industry supply chain, firstly this paper defines the supply chain risk management problem in Chinese chemical industry. Then this paper develops a stochastic chance-constrained programming model to select optimal mitigation strategies effectively to achieve risk reduction goals without exceeding the expenses budget. Finally, this paper gives a numerical example and discusses how the change of model parameter affects the optimal solution.
\end{abstract}

Keywords: Chemical industry supply chain risk risk management; stochastic chance-constrained programming model

\section{Introduction}

Over the last 10 years, earthquakes, economic crises, SARS, strikes, terrorist attacks have disrupted supply chain operations repeatedly. Supply chain risks can have significant impact on a firm's short-term performance. For example, Ericsson lost 400 million Euros after their supplier's semiconductor plant caught on fire in 2000, and Apple lost many customer orders during a supply shortage of DRAM chips after an earthquake hit Taiwan in 1999. Supply chain risks can have long-term negative effects on a firm's financial performance as well. For instance, companies suffering from supply chain risks experienced 33-40\% lower stock returns relative to their industry bench- marks. To mitigate supply chain risks associated with various types of risks (uncertain economic cycles, uncertain consumer demands, and unpredictable natural and man-made disasters), many researchers have developed different strategies/models for managing supply chain risks.

While globalization is the mainstream of the world, supply chains are gradually vulnerable. Supply chains face both internal risks and external risks. This affects chemical industry supply chain to operate continuously and safely. Especially the chemical and related chemical industry are particularly exposed to high environmentally related costs arising from normal operation and accidents, not only of their own processes but also other processes in their supply chain. So it is necessary to research on how to manage risks in chemical industry supply chain and help chemical industry operate safely.

Ram Narasimhan and Srinivas Talluri (2009) give perspectives on risk management in supply chains [1]. Mihalis Giannakis and Michalis Louis (2011) present a multi-agent based framework for supply chain risk management [2]. Fernandes et al. (2010) construct a risk management framework for the petroleum supply chain [3].

There are some methods for supply chain risk management, such as generic prescriptive methodology [4], a minimal agency model [5], maintenance-based strategies [6], a robust optimization approach [7]. Nagurney, A. (2005) considers the dynamics of a global supply chain network economy in the presence of risk and uncertainty and a multi-criteria decision-making problem [8]. Cheng et al. (2012) conduct a survey study in the Chinese business context. It investigates the importance for purchasing firms forming guanxi networks with key suppliers in perceiving supply risk. Using a food supply chain as a case [18]. Chen (2012) applies the option

\footnotetext{
*Corresponding author e-mail: sxli@ecust.edu.cn
} 
theory to investigate the risk cash flows in a supplier-retailer channel. When determining the investment threshold value, the members of a supply chain should consider the volatility of demand shock, sunk cost, shipping costs, holding costs. This option game model seems to be a popular model [19]. G.E. Applequist et al. (2000) adopt an effective polytope integration method for evaluation of expected values and variances of revenue which can account for the effects of demand uncertainties on revenue while recognizing the uncertainty in inventory over time [20]. The combination of these elements with conventional deterministic mathematical programming models offers the promise of providing an effective approach to accommodating uncertainties and a rational basis for balancing risk [21].

As far as chemical industry is concerned, an approach using new concepts for risk management of chemical supply chain investments [20]. Li and Peng give a risk assessment indicator system combining logic inference, Delphi method and AHP [17]. Faisal I. Khan and S. A. Abbasi (1998) describe a new software package for conducting rapid risk assessment (RRA) in chemical process industries [10]. Berning et al. (2004) consider a complex scheduling problem and supply chain optimization in the chemical process industry [11].

Though there are not a few literatures focus on supply chain risk management, there are few literatures considering stochastic risk management in the process of risk strategies selection. In this paper, according to the features of chemical industry supply chain risks, a stochastic chance-constrained model is developed to ensure risk mitigation level in chemical industry supply chain.

This paper is organized as fowling. The second section is problem definition, in which representative risks and mitigation strategies in Chinese chemical industry supply chain is given, then risk mitigation strategies selection problem is presented. The third section is the development of stochastic chance-constrained programming model. The fourth section is numerical examples. Finally conclusion of this paper is given and future works are presented.

\section{Problem Definition}

There are some representative risks and mitigation strategies in Chinese chemical industry supply chain as the following:

(1) Supply risk: improper selection of supplier or deficiency of supplier' capability. Supply risk is perceived by the effect that purchased items and services have on corporate profitability, market factors, and supplier characteristics.

(2) Capacity risk: lack of flexibility. The impact of mass retirement on your firm's ability to make a product or provide a service.
(3) Environment risk: accident and pollution [13]. Environment risk is conceptualized as the impact of the firm's external environment on the firm from a cash flow and profitability perspective. Environment Risk is viewed as the probability that a hazard will occur during a particular time period.

(4) Disruption risk: natural disaster, war and terrorism etc. Disruption risk has received increasing attention in the last few years, when it comes to global supply chains, the potential for disruption comes in many packages, from large-scale natural disasters and terrorist attacks to plant manufacturing fires, widespread electrical blackouts, and operational contingencies such as shipping ports too small to handle the flow of goods coming into a country.

(5) Equipment failure: improper of equipment maintenance, improper operation.

(6) Delay risk: Inflexibility of supply source, failure of production control etc. Delay risk accounted for $4.58 \%$ of the total variance and ranked first with an importance index of 79.00. This factor contained a combination of two variables: construction delay and third parties' delay. Both variables had a relatively high importance index of 82.48 (construction delay) and 75.64 (third parties' delay). Construction and third party delay are the two most important components of delay risk. Failure to operate the underground rail system within scheduled time was attributed to these two variables of delay risk and reflected in the following statements.

(7) Inventory risk: improper inventory [14]. Excess inventory hurts financial performance. That was the case in late 2000, when the personal computer industry carried roughly 12 weeks of inventory. The killer combination of excess inventory and falling prices hurt many companies, notably Compaq Computer Corp. Inventory risk hinges on three factors: the value of the product, its rate of obsolescence and uncertainty of demand and supply. As we have seen, holding excess inventory for products with high value or short life cycles can get expensive. The strategy can work quite well, however, for low-value commodity products that have low obsolescence rates. To complicate matters even Risk mitigation involves the adoption of a safety culture and a number of organizational strategies which can affect and limit risk. And the mitigation strategies include: The application of three key risk/reward relationships in the supply chain suggests four general strategic approaches, depending upon the relative level of risk and the cost of mitigating it.

(8) Forecast Risk: Forecast risk results from a mismatch between a company's projections and actual demand. If forecasts are too low, products might not be available to sell. Forecasts that are too high result in excess inventories and, inevitably, price mark downs. Long lead times, seasonal demand, high product variety and smaller product life cycles all increase 
forecast error. Errors tend to be greater when a few customers make larger purchases (as opposed to many customers making smaller purchases).

Forecast inaccuracies can also result from information distortion within the supply chain. In late 2003, for example, product shortages in Western Europe led Nokia customers to order more than they needed so they would be able to meet demand if Nokia began rationing or allocations. Unfortunately, the exaggerated figures distorted Nokia's reading of the market, causing the company to inaccurately forecast sales.

Other causes of information distortion include promotions and incentives that lead to forward buying; batching of purchases, which leads to higher volatility in orders; and lack of knowledge of end-customer demand at upstream locations. Distortion increases in the supply chain as you get farther away from.

And the mitigation strategies include:

(1) Add capacity: capacity reservation or purchasing from outside.

It means focusing on low-cost, decentralized capacity for predictable demand, building centralized capacity for unpredictable demand and increasing decentralization as cost of capacity drops.

When capacity is expensive, managers can reduce supply chain costs by centralizing capacity to pool risk. As costs decline, capacity must be decentralized further. Consider the personal computer industry. PCs can be assembled to order in two different ways. In one, the Dell model, capacity is central-zed. In the other model, widely used in India, several companies sell component kits to local assemblers for assembly on demand. Given the low cost of assembly capacity in India, it is economical to decentralize capacity, even though this action educes pooling and increases the overall size of assembly capacity across the supply chain. In contrast, given the higher cost of capacity in the United States, centralizing buffer capacity is more effective.

(2) Have redundant suppliers: it can reduce the dependence of single supplier and present alternative in abrupt incidents. It is effective to mitigate supply, disruption and delay risk.

(3) Safe management: It mainly adopts the conception of safe production. It is very effective to mitigate environment risk [15].

(4) Increase flexibility: flexibility of production process. It Favors cost over flexibility for predictable, high-volume products.

Also, needs flexibility for low-volume unpredictable products.

Centralize flexibility in a few locations if it is expensive.

(5) Information management strategies: reducing the standard deviation of the demand over the replenishment lead time would result in inventory reduction for the entire supply chain. When managing products with short life cycles, short replenishment lead times could enable a retailer to place more than one order over the selling season.

(6) Increase inventory: Decentralize inventory of predictable, lower-value products.

Centralize inventory of less predictable, higher-value products.

(7) Robust strategies: the strategy would enable a firm to manage operational risks efficiently regardless of the occurrence of major disruptions; the strategy would enable a firm to sustain its operation during a major disruption and recover quickly after a major disruption [12].

Different mitigation strategy has different effect on one risk, it is necessary to access how much different mitigation strategy affects different risk.

There is definition of Table 1 .

Let $a_{i j}(i=1,2, \cdots, m ; j=1,2, \cdots, n)$ be risk reduction proportion that mitigation strategy $j$ acts on risk $i$.

Let $u_{i}\left(u_{i} \sim N\left(\mu, \sigma^{2}\right)\right)$ be the risk $i$ reduction proportion that should be achieved at least. It is assumed to be random and normally distributed.

Let $c_{j}$ be the cost of mitigation strategy $j$.

Companies mostly aim to achieve more money with less cost, and they will not put too much money to mitigate risks.

Let $t$ be the total money that company would devote to mitigation strategies.

Table 1: Risks and mitigation strategies

\begin{tabular}{lccccc}
\hline & $c_{1}$ & $c_{2}$ & $\ldots$ & $c_{n}$ & $t$ \\
& Stra.1 & Stra.2 & $\ldots$ & Stra.n & \\
\hline Risk 1 & $a_{11}$ & $a_{12}$ & $\ldots$ & $a_{1 n}$ & $u_{1}$ \\
Risk 2 & $a_{21}$ & $a_{22}$ & $\ldots$ & $a_{2 n}$ & $u_{2}$ \\
$\ldots$ & $\ldots$ & $\ldots$ & $\ldots$ & $\ldots$ & $\ldots$ \\
Risk $m$ & $a_{m 1}$ & $a_{m 2}$ & $\ldots$ & $a_{m n}$ & $u_{m}$ \\
\hline
\end{tabular}

When more mitigation strategies are taken up, the risk reduction proportion will improve but cost of mitigation strategies will increase. So it is necessary to find a solution that can achieve risk reduction goals and cost least together. That is how to select strategies from strategy 1 to strategy $n$.

\section{The Model of Supply Chain Risk based on Stochastic Chance-constrained Programming Model}

If the strategy cost of risk reduction exceeds the benefits of risk reduction, companies will have no desire to mitigate risks actively. Which strategy should be selected?

Let $x_{j}$ express that whether mitigation strategy $j$ is adopted. The value of $x_{j}$ is only 0 or 1 . If $x_{j}=1$, it means mitigation strategy $j$ is adopted; If $x_{j}=0$, it means mitigation strategy $j$ is not adopted. $a_{i j}$ means risk 
reduction proportion that strategy $\mathrm{j}$ acts on risk $i$. Of course, total risk reduction proportion that all strategies act on risk $i$ is $\sum_{j=1}^{n} a_{i j} x_{j}$. So total risk reduction proportion that all strategies act on all risk is $\sum_{i=1}^{m} \sum_{j=1}^{n} a_{i j} x_{j}$.

The goal of risk management is to reduce risk as much as possible. So the objective function is to maximize total risk reduction proportion that all strategies act on all risks as shown in Equation (1):

$$
\max z=\sum_{i=1}^{m} \sum_{j=1}^{n} a_{i j} x_{j}
$$

The cost of strategy $j$ is $c_{j}$, and total cost of all Strategies is $\sum_{j=1}^{n} c_{j} x_{j}$. However, the total money that company would devote to risk management is $t$. Accordingly, constraint condition (2) exists:

$$
\sum_{j=1}^{n} c_{j} x_{j} \leqslant t
$$

Reduction proportion of risk $i(i=1,2, \ldots, m)$ is assumed not less than $u_{i} . u_{i}$ is assumed to be random and normally distributed. Suppose significance level is $\alpha_{i}$. Therefore another constraint condition (3) exists:

$$
P\left(\sum_{j=1}^{n} a_{i j} x_{j} \geqslant u_{i}\right) \geqslant \alpha_{i} \quad i=1,2, \cdots, m
$$

According to former analysis, we can develop a stochastic chance-constrained programming model to solve the problem above as shown in (4).

$$
\begin{aligned}
& \max z=\sum_{j=1}^{n} \sum_{i=1}^{m} a_{i j} x_{j} \\
& \text { s.t. }\left\{\begin{array}{l}
P\left(\sum_{j=1}^{n} a_{i j} x_{j} \geqslant u_{i}\right) \geqslant \alpha_{i} \quad i=1,2, \cdots, m \\
\sum_{j=1}^{n} c_{j} x_{j} \leqslant t \\
x_{j}=0, \text { or } 1 \quad j=1,2, \cdots, n
\end{array}\right.
\end{aligned}
$$

The objective function is to achieve the maximal total risk reduction proportion. The first constraint condition means the probability of every risk reduction proportion should not be under the base line $u_{i}$ is not less than $\alpha_{i}$. The second constraint denotes that the cost of mitigation strategies should not exceed the total money $t$.

Baoding Liu et al. (2005) give the method of solving the stochastic chance-constrained programming model [16]. Likely, (3) can be changed to (5).

$$
\sum_{j=1}^{n} a_{i j} x_{j} \geqslant K_{\alpha_{i}}, i=1,2, \cdots, m
$$

And

$$
K_{\alpha_{i}}=\inf \left\{K \mid K=\phi^{-1}\left(\alpha_{i}\right)\right\}, i=1,2, \cdots, m
$$

Hence the stochastic chance-constrained programming model shown in (4) can be changed to (7).

$$
\begin{aligned}
& \max z=\sum_{j=1}^{n} \sum_{i=1}^{m} a_{i j} x_{j} \\
& \text { s.t. }\left\{\begin{array}{l}
\sum_{j=1}^{n} a_{i j} x_{j} \geqslant K_{\alpha_{i}}, i=1,2, \cdots, m \\
\sum_{j=1}^{n} c_{j} x_{j} \leqslant t \\
x_{j}=0, \text { or } 1 j=1,2, \cdots, n
\end{array}\right.
\end{aligned}
$$

Software Lingo can be used to solve (7).

\section{Numerical example}

A numerical example is given here (see Table 2).

Suppose $u_{i} \sim N\left(1,2^{2}\right)$.

Supposed there exist six risks (information risk, capacity risk, green risk, partnership risk, transportation risk and control risk) in chemical industry supply chain. Moreover, there are five risk mitigation strategies (add capacity, information share, safety management, increase flexibility and robust planning). Figures in Table 2 denote risk reduction proportion that particular mitigation strategy acts on particular risk. For example, risk reduction proportion of Add capacity acts on Partnership risk is 10 . The cost of every mitigation strategy is given on the base line.

The optimal solution is that the company should adopt three mitigation strategies (add capacity, Increase flexibility, Robust planning). It can reduce risks to particular extent and will not go beyond the budget of money. The conclusion can help chemical industry manage supply chain risks by scientific method.

From above it shows that the value changes of $a_{i j}, u_{i}, c_{j}$ and $t$ will affect the optimal solution (i.e. how to select mitigation strategies).

Firstly, $a_{i j}$ is different for different chemical industry supply chain. For example, Increase flexibility may be effective mitigation strategy for capacity risk in daily chemical industry supply chain but not so effective in pharmacy industry.

Secondly, there is different requirement of $u_{i}$ in different chemical industry. For example, the hazardous chemical industry may care about environment risk more than daily chemical industry and it need a high value of $u_{i}$ (i.e. it may want to mitigate environment risk more than other industry).

Finally, the value of $t$ depends on the company leaders' consideration and the cost of risk reduction. For instance, if the company leader is aware of the importance of risk reduction, he will devote more money to do it.

\section{Conclusion and Future Works}

In this paper, we deduce generic risks and mitigation strategy in chemical industry supply chain after related 
Table 2: Numerical example

\begin{tabular}{lcccccc}
\hline Risk Strategy & $\begin{array}{c}\text { Add } \\
\text { cap. }\end{array}$ & $\begin{array}{c}\text { Inf. } \\
\text { Shr. }\end{array}$ & $\begin{array}{c}\text { Saf. } \\
\text { Mgmt }\end{array}$ & $\begin{array}{c}\text { Inc. } \\
\text { Flex. }\end{array}$ & $\begin{array}{c}\text { Rob. } \\
\text { plan. }\end{array}$ & $\alpha_{i}$ \\
\hline Inf. risk & 5 & 20 & 0 & 10 & 15 & 0.7 \\
Cap. risk & 25 & 5 & 0 & 15 & 10 & 0.8 \\
Green risk & 5 & 0 & 25 & 10 & 20 & 0.9 \\
Part. risk & 10 & 20 & 15 & 15 & 25 & 0.7 \\
Transp.isk & 15 & 0 & 10 & 10 & 20 & 0.9 \\
Other risk & 25 & 20 & 5 & 10 & 15 & 0.6 \\
cost & 10 & 20 & 5 & 8 & 12 & \\
\hline
\end{tabular}

literature review. In order to select optimal mitigation strategies for achieving risk reduction goals without exceeding the expenses budget, we develop a stochastic chance-constrained programming model. Then we present a numerical example. Finally we discuss how model parameter change affects the optimal solution.

Future work may contain there aspects:

Firstly, supply chain risk management of hazardous chemicals industry is worthy to be focused on.

Secondly, it is very meaningful to give the probability of risks and quantity the consequence of risks.

Finally, we supposed various risks are the same important for company to mitigate. However, the risks importance ranking will be different for different chemical industries or companies. So it is necessary to introduce priority coefficient into the objective function as far as different chemical industry is concerned.

\section{Acknowledgement}

This research was supported by the National Natural Science Foundation of China (NSFC) for the projects of "Transportation mode and transportation route optimization of hazardous materials multimodal transportation based on dynamic risk (No. 71302043), "Dynamic Environmental Risk Assessment and its Evolution Forecast of Hazardous Chemicals Accidents" (No. 71001039), "Cross-docking-based Scheduling Optimization Method for Emergency supplies" (No. 71101051) and "Production Strategy and Scheduling Problem for Hybrid Production System with Outsourcing Allowed" (No. 71202053), and also by Humanities and Social Sciences Youth Fund from Ministry of Education for the project of "Research on dynamic risk assessment for transport of dangerous chemicals" (No. 12YJC630120) and National Key Technology R\&D Program "R\&D and Application Demonstration of Supply Chain Collaborative E-commerce Technology" (No. 2013BAH11F00).

\section{References}

[1] R. Narasimhan, S. Talluri, Journal of Operations Management, 27, 114-118 (2009).
[2] M. Giannakis, M. Louis, Journal of Purchasing and Supply Management, 17, 23-31 (2011).

[3] J.Leão. Fernandes, A.P.Barbosa-Póvoa and S.Relvas, Computer Aided Chemical Engineering, 2, 157-162 (2010).

[4] P. R.Sinha, L. E.Whitman, D.Malzahn, Supply Chain Management-An International Journal, 9, 154-168 (2004).

[5] P. J.Agrell, R. Lindroth, A., Norrman, International Journal of Production Economics, 90, 1-16 (2004).

[6] C. G. Vassiliadis and E. N. Pistikopoulos, Journal of Hazardous Materials, 71, 481-501 (2000)

[7] G. J. Hahn, H. Kuhn, International Journal of Production Economics, 139, 135-144 (2012).

[8] A.Nagurney, D.Matsypura, Transportation Research and Elogistics and Transportation Review, 41, 585-612 (2005).

[9] G. E. Applequist, J. F. Pekny, G. V. Reklaitis, Computers and Chemical Engineering, 24, 2211-2222 (2000).

[10] F. I. Khan and S. A. Abbasi, Environmental Modelling and Software, 14, 11-25 (1998).

[11] G,Berning, M.Brandenburg, K.Gürsoy, Jürgen, S. Kussi, V.Mehta and F.J. Tölle, Computers \& Chemical Engineering, 28, 913-927 (2004).

[12] S. T. Christopher, International Journal of Production Economics, 103, 451-488 (2006).

[13] L. P. Liu, S. H. Liu, S. X. Li, Advanced Materials Research, 328-330, 751-754 (2011).

[14] G. P. Cachon, Management Science,50, 222-238 (2004).

[15] L. P. Liu, Shanghai Jiao Tong University press, April, (2010).

[16] B. D. Liu et al., Tsinghua University Press, (2005).

[17] Li, M. L., H. J. Peng, et al., Applied Mathematics \& Information Sciences, 6, 9-14 (2012).

[18] Cheng, T. C. E., Yip, F. K., Yeung, C. L., International Journal of Production Economics, 139, 3-13 (2012).

[19] Chen, P.-Y., International Journal of Production Economics, 139, 80-89 (2012).

[20] G. E. Applequist, J. F. Pekny, G. V., Reklaitis Computers \& Chemical Engineering, 9-10, 2211-2222 (2000).

[21] Sunil Chopra, M. S. Sodhi, MIT Sloan Management Review, 1, 53-62 (2004).

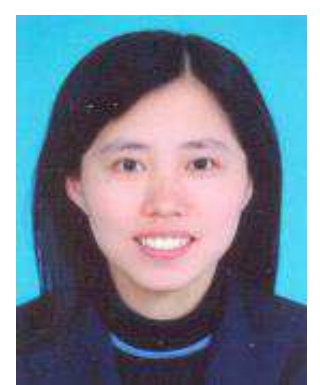

Liping Liu is Associate Professor of East China University of Science \& Technology in China, got her Ph.D. of Management at Shanghai Jiaotong University of China in 2008. Her research interests include risk management, optimization method and supply chain management. 


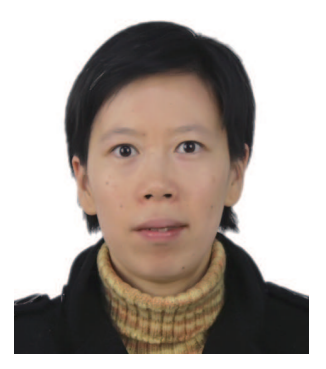

assessment, scheduling management.
Shuxia Li is Associate Professor of East China University of Science \& Technology in China. She received the $\mathrm{PhD}$ degree in Mechanical and Electronic Engineering at Huazhong University of Science \& Technology in China. Her research interests include risk optimization and operations

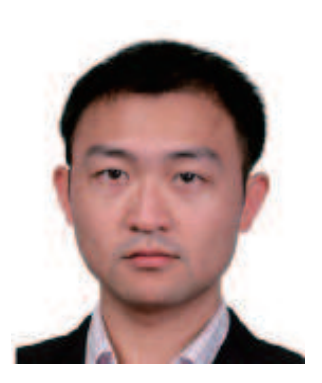

Yifan $\mathbf{W u}$ is an associate professor in School of Business, East China University of Science and Technology. He received his $\mathrm{BE}$ and $\mathrm{PhD}$ in Industrial Engineering and Management from Shanghai Jiao Tong University in 2004 and 2010, respectively. His current research interests include supply chain management under disruption risk, logistics system optimisation and general operations and supply chain management. 